\title{
Microstructural characterization of Inconel X750 alloy used in reactors components.
}

Ana Lucía Marzocca ${ }^{1 *}$, Patricia Bozzano ${ }^{1}$, Juan Nervi ${ }^{2}$

${ }^{1}$ Constituyentes Atomic Center of National Commission of Atomic Energy (CAC-CNEA), Electron Microscopy Division, San Martín, Buenos Aires, Argentina.

${ }^{2}$ Materials and Micromechanics Division Head, Nucleoeléctrica Argentina S. A., CABA, Argentina. *Corresponding author: marzocca@cnea.gov.ar.

Incone ${ }^{\circledR}($ Inconel is a registered trademark of Special Metals Corporation and its subsidiaries) X-750, is an alloy Ni-based which was thermally treated to create a high density of precipitates (precipitationhardened). It's composed at the same time with the following elements: $\mathrm{Cr}, \mathrm{Ti}, \mathrm{Al}$ and $\mathrm{Nb}$. These alloys were developed for high temperature applications, and are required to have high resistance to corrosion and mechanics [1,2].

The objective of this work is the complete characterization of the microstructure using microscopy techniques. In this research paper will presents the first results obtained from the study of this material by means of optical microscopy (OM) and scanning electron microscopy (SEM).

The sample preparation consisted of polishing with diamond paste up to $1 \mathrm{um}$, then a chemical attack was carried out with Kalling $\left(\mathrm{CuCl}_{2}\right.$ in $\mathrm{HCl}$ and ethanol) reagent to reveal the microstructure [3].

Through observation with the OM, a microstructure of equiaxiated grains of heterogeneous sizes was determined. It was also observed the presence of precipitates of different sizes, shapes and colors ("pink": those of stable form and "gray": those of more elongated shape) distributed by the matrix and at the edges of grain.

They were observed and analyzed in the FEI SEM Quanta 200 equipped with EDAX Apollo belonging to the Laboratory of Electron Microscopy of the Materials Management in Constituyentes Atomic Center of National Commission of Atomic Energy, CAC - CNEA. In Figure 1, the precipitate has a square shape, the major element is $\mathrm{Ni}$ (73\% by weight); while the precipitate in Figure 2 has a high percentage of $\mathrm{Nb}$ (65\% by weight) and Ti.

As a conclusion of this first characterization: the sample has precipitates of $\mathrm{Ni}$ and $\mathrm{Nb}$. We found, in smaller quantity, heterogeneous precipitates with dark areas inside and with a variation in the composition respect to another zone of the same precipitate [4]. 


\section{References:}

[1] C. D. Judge et al., Proceedings of the $18^{\text {th }}$ International Conference on Environmental Degradation of Materials in Nuclear Power Systems (2018) p. 727-740.

[2] C.D. Judge et al Journal of Nuclear Materials 492 (2017), p. 213-226.

[3] M. Miyagusuku et al., Book of extended Abstracts of SAM-CONAMET (2018) p. 1296-1298.

[4] G. Zbihlei and R. Castillo Guerra from Materials Management-CNEA are thanked for their help in the preparation of material for observation.

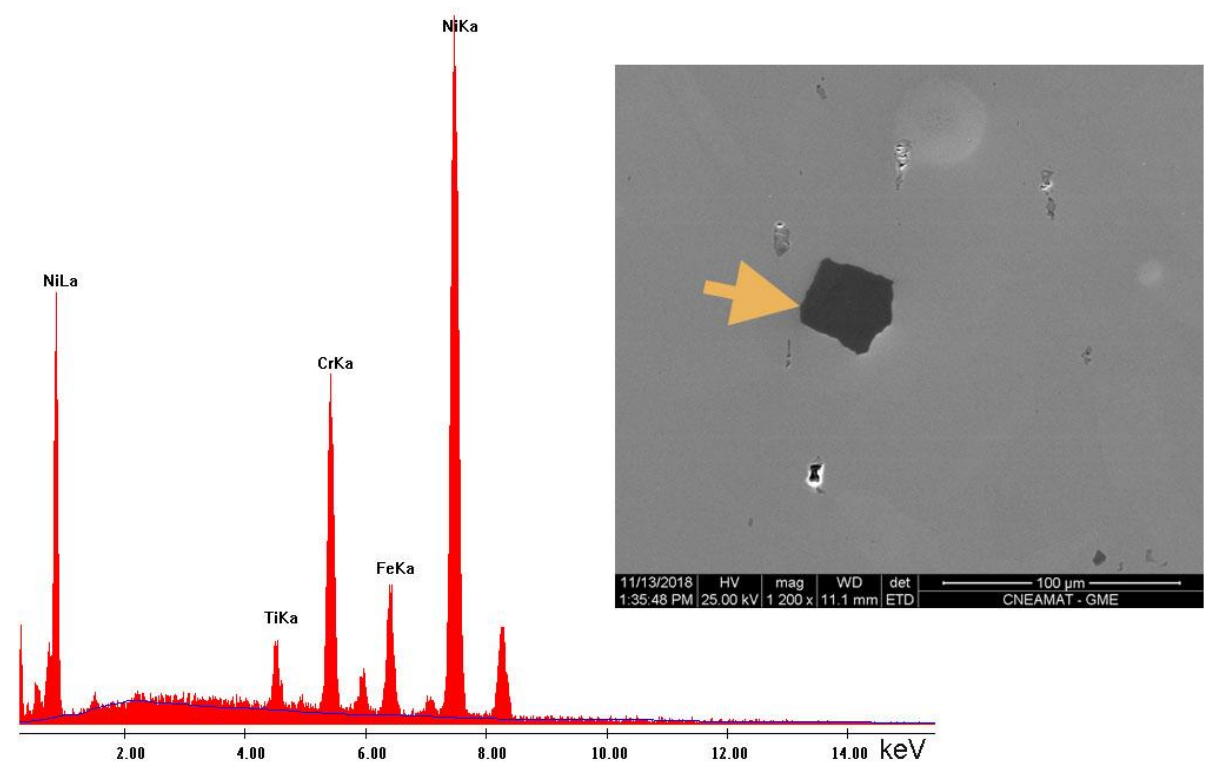

Figure 1: SEM micrograph (right) and EDS spectrum (left) of the precipitate with a square shape marked with an arrow.

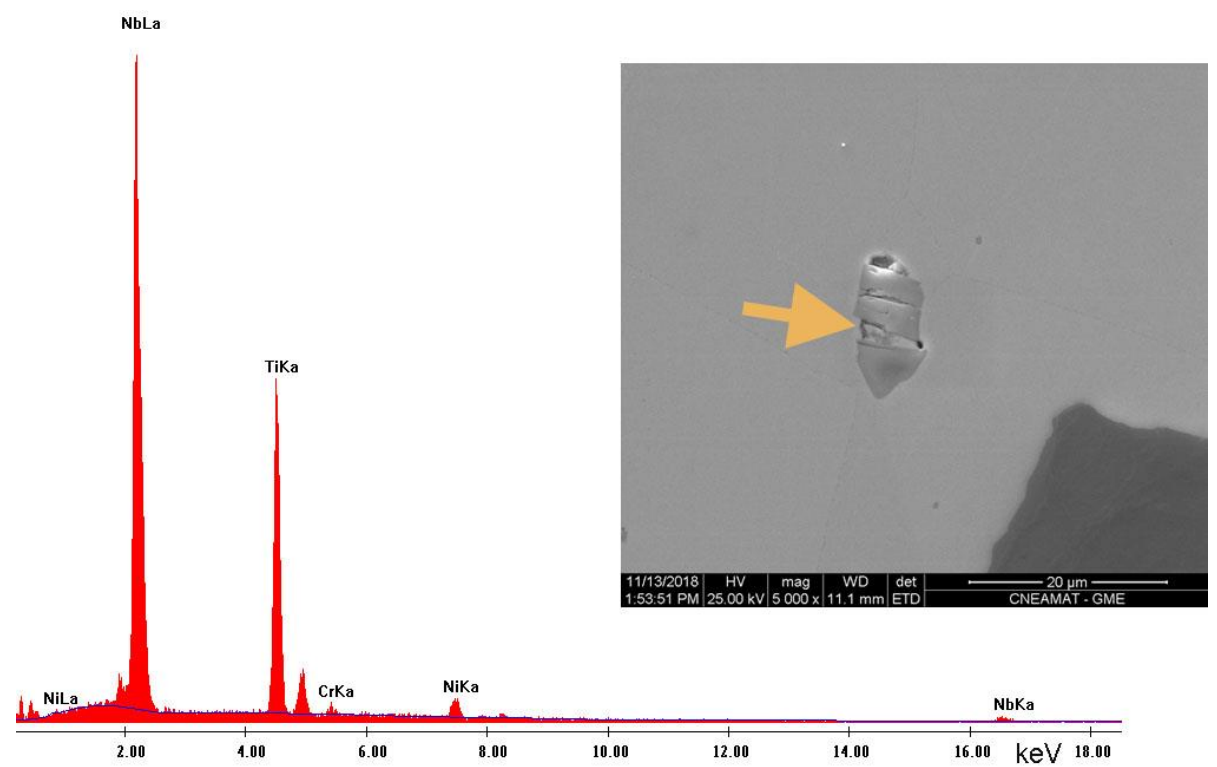

Figure 2: SEM micrograph (right) and EDS spectrum (left) of the precipitate marked with an arrow. 